\title{
CLOSED LOOP CONTROL TECHNIQUES FOR THE GROWTH OF SINGLE CRYSTAL TURBINE COMPONENTS
}

\author{
M. Eric Schlienger \\ Sandia National Laboratories \\ Albuquerque, NM
}

\begin{abstract}
Analysis of processes used for the production of single crystal turbine components reveals significant shortcomings. Inadequate consideration has been madc of the fact the system is cooling dominated and that the amount of cooling tends to increase as the emissive cooling area expands during the process. Experimental evidence suggests that during processing, this increased cooling causes the solidification interface to move away from the baffle and become curved. The motion of the interface results in a decrease in the solidification gradient. The combination of these actions can result in variations in PDAS, grain misalignment and the production of defects. It is shown that despite this tendency, microstructural stabilization may be achieved through the use of the heat of fusion as an internal process heat source. ${ }^{1}$
\end{abstract}

\section{Introduction}

Directionally solidified and single crystal turbine components are seeing increased utilization in both aviation and land based power turbines ${ }^{2}$. Due to the complexity of the process, production of these parts is an expensive and time consuming operation. The process for any given part often entails a withdrawal cycle of several hours, during which the single crystal parts are grown. When the mold expense is combined with the processing costs associated with the casting and growth of these components, such parts represent a substantial investment as they emerge from the furnace. Therefore, casting or solidification defects which impact product yield represent a considerable expense, lowering margins and raising cost. Further, concern has been voiced that with the continued expansion of the use of DS \& SX parts, the application of these parts may soon become foundry capacity limited. The combination of the capacity and expense issues provides a driving force for the development of techniques that improve process yield and throughput. It is towards this end that this work explores processing techniques and the single crystal growth process as it occurs in industrial furnaces.

\section{Solidification Apparatus}

For this work, single crystal test bars of CMSX-4 were Superalloys 1996

Edited by R. D. Kissinger, D. J. Deye, L. L. Anton,

A. D. Cetel, M. V. Nathal, T. M. Pollock, and D. A. Wood ford

The Minerals, Metals \& Materials Society, 1996 produced in ten different process cycles under varying conditions. The test bars were produced in a new single crystal furnace manufactured by Retech Inc. and being built for Rolls Royce. The furnace is of a modified Bridgeman design ${ }^{3}$, the solidificalion portion is shown schematically in Figure 1.

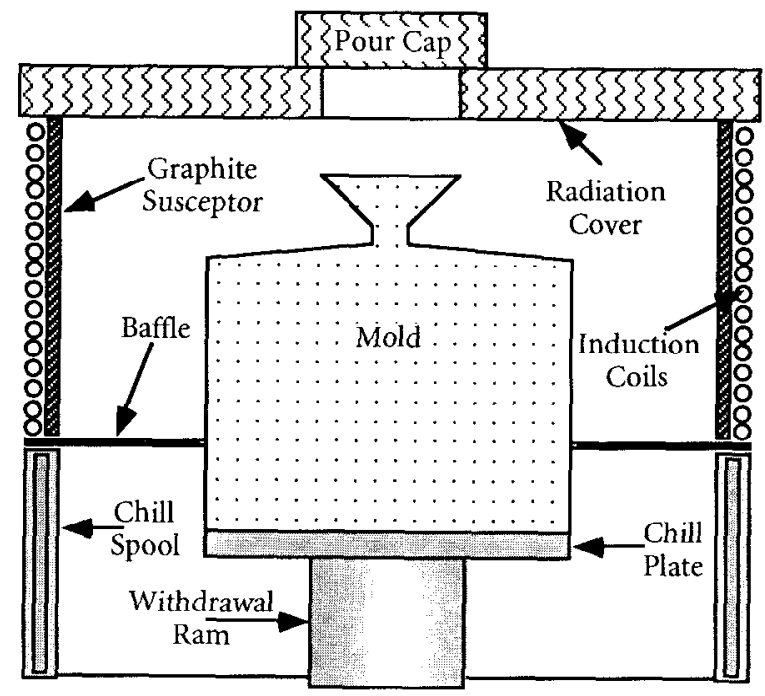

Figure 1: Typical configuration of single crystal turbine blade solidification apparatus.

During processing, metal is melted in an induction crucible. Once the metal has been heated to the appropriate temperature, the pour cap is removed and the metal is poured into the pre-heated mold. The mold was heated, prior to and during the solidification process, by an inductively heated graphite susceptor. The pour cap is replaced immediately after pouring. The mold, which is placed on a water cooled chill plate, is held in place after the pour for a span of time sufficient to allow dendritic solidification to become established as a result of the heat flux through the molten metal and into the chill plate. During this period, a crystal selector 4 inhibits slower growing dendrites such that only a single dendrite orientation is allowed to propagate through the part. After the hold time, the withdrawal process is initiated and the mold is lowered in a controlled fashion. As the mold is moved past the baffle, it is subjected to a temperature 
gradient intended to maintain dendritic growth and the single crystal morphology which has been established.

\section{Current Control Techniques}

Numerous articles have been written on the proper technique for the growth of single crystal turbine blades 5,6 . Perhaps the most prevalent control mechanism that has been proposed is expressed by the equation 78 :

$$
k=\frac{G}{R}
$$

Where $\mathrm{k}$ is a constant dependent on the alloy, $\mathrm{G}$ is the gradient at the solidification front and $R$ is the growth rate. The ratio $G / R$ is regularly used to describe the solidification environment. It implies that optimum crystal growth will occur when $G$ and $R$ are varied in such a fashion as to keep the above relationship equal to a constant. In practice this requires measuring the gradient and solidification rate on a continuous basis. Past implementations have made the simplifying assumption that the solidification rate is equivalent to the withdrawal rate. Unfortunately this is only true in a macroscopic sense and does not take into account the effects of the rapid geometrical changes that are typical of turbine blades.

A more typical process involves a mold heater which is held at a constant temperature, and the parts are withdrawn from the hot zone of the furnace to the cold, at a constant velocity. A variation of this technique involves modification of the withdrawal velocity to accommodate geometrical features of the part.

\section{Analysis of $G / R$ Techniques}

To evaluate the basis of (1), the solidification phenomena that occur at the liquid solid interface must be examined. After the work of Kurz and Fisher ${ }^{9}$, Fick's second law may be re-written for a steady state, moving reference frame as:

$$
\frac{\partial^{2} C}{\partial z^{2}}=\frac{-V}{D} \frac{\partial C}{\partial z}
$$

Where $C$ is the solute concentration in the liquid, $z$ is the distance in front of the solidification interface, $V$ is the solidification velocity and $\mathrm{D}$ is the solute diffusivity in the liquid. The solution to this differential equation is of the form:

$$
C=A+B e^{-\left(\frac{V_{z}}{D}\right)}
$$

In order to evaluate the boundary conditions which will yield a complete solution, a further simplification is made. For the purposes of this analysis, it is assumed that the diffusion of solute in the solid material is very slow when compared to the solidification rate and the liquid diffusion rate. In fact, the difference in the solute diffusion rates of the liquid to the solid is typically on the order of 3 to 4 orders of magnitude and therefore for the purpose of this analysis it may be assumed that there is: no compositional variation in the solid past the initial transient. With this assumption in mind, the solution is therefore only valid for positive values of $z$, that is to say, within the liquid.

In order to obtain the complete solution, A and B in (3) must be evaluated. This is achieved by examination of the boundary conditions. Assuming eutectic solidification, and liquid of nominal composition $\mathrm{C}_{0}$, steady state solidification must ultimately produce a solid of the same composition. As such, a solid of composition $\mathrm{C}_{0}$ must be forming from a liquid of composition $\mathrm{C}_{0} / \mathrm{k}$. There is then a boundary layer in advance of the interface into which solute is being rejected such that ultimately, at the solidification interface itself the composition of the liquid is $\mathrm{C}_{0} / \mathrm{k}$. Evaluation of the boundary conditions yields the complete expression for the composition of the liquid as a function of the distance in front of the solidification interface:

$$
C=C_{0}+\left(\frac{C_{0}}{k}-C_{0}\right) e^{-\left(\frac{V_{z}}{D}\right)}
$$

Equation 4 implies that there is an exponentially decreasing solute concentration gradient in advance of the solidification interface. If the liquidus temperature is assumed to decrease with composition, then the liquidus temperature of the alloy within the diffusion boundary layer increases in a logarithmic fashion at positions away from the solidification interface.

For solidification to occur, heat must be extracted from the system. In the case of the growth of single crystals, as described, the heat flux is in the $-z$ direction. Since the alloy has a finite thermal conductivity, this flux creates a gradient in the material through which it flows. It is possible that for the liquid in advance of the solidification front, the liquidus temperature, due to the compositional variation, may increase more rapidly than the liquid metal temperature increase that occurs as a result of the temperature gradient. This situation results in a region of liquid metal which is undercooled with respect to the local liquidus temperature in front of the solidification interface. This phenomena is termed constitutional undercooling. Convex perturbations of the solid / liquid interface into the molten metal can extend rapidly under these conditions. This extension occurs because the perturbation is growing into a region where the interface is increasingly undercooled with respect to the local liquidus temperature. Therefore if a planar interface is assumed, then in order to ensure that a planar interface remains stable, the temperature gradient in the region of the solidification front must be sufficiently high to preclude any constitutional undercooling.

In order to evaluate the temperature gradient which must be maintained to preclude interface instabilities, it is first necessary to obtain an expression for the liquidus temperature in advance of the interface. It may be shown that the expression for the liquidus temperature in advance of the interface is:

$$
T_{L}=T_{L\left(C_{0}\right)}-\left(T_{L\left(C_{0}\right)}-T_{S\left(C_{0}\right)}\right) e^{-\left(\frac{V_{z}}{D}\right)}
$$

In addition, the expression for the temperature of the molten metal in advance of the interface, $T_{q}$, is a function 
of the gradient and written as:

$$
T_{q}=T_{s\left(c_{0}\right)}+G z
$$

Equation (7), which is the difference between (5) and (6), therefore describes the undercooling of the liquid metal as a function of the distance in advance of the interface.

$$
T_{U}=\left(T_{L\left(C_{0}\right)}-T_{s\left(C_{0}\right)}\right)\left(1-e^{-\left(\frac{V_{z}}{D}\right)}\right)-G z
$$

It is reasonable to argue that if there is no position in advance of the interface where the liquid metal is undercooled, then a planar interface will be stable. If (7) is differentiated the result represents the slope of the undercooling function; if at all times this slope is less than zero, then none of the liquid will be undercooled.

$$
\frac{d T_{t}}{d z}=\frac{\left(T_{L\left(C_{0}\right)}-T_{S\left(C_{0}\right)}\right) V}{D} e^{-\left(\frac{V z}{D}\right)}-G
$$

The exponential in (8) has its maximum value at $z=0$. It may therefore be written that the condition for the stability of a planar interface is:

$$
G \geq \frac{\left(T_{L\left(C_{0}\right)}-T_{S\left(C_{0}\right)}\right) V}{D}
$$

This necessarily neglects the fact that the suppression of the melting point that occurs due to the curvature of an interface can have a local stabilizing effect that will allow some perturbations to exist ${ }^{10}$. however for practical processing conditions, such perturbations are not significant. This equation may be re-written by combining the alloy dependent values of $T_{L}$ and $T_{S}$ into a single constant $K$. The result, equation (10), is in fact the same as (1) with the only difference being that the interface velocity $\mathrm{V}$ has been used instead of the solidification rate R.

$$
K=\frac{G}{V}
$$

As an example, the gradient required to insure a planar solidification in an Aluminum alloy with 2.5 atomic percent copper is calculated. This system is used because it is well characterized. Given a diffusivity of $.003 \mathrm{~mm}^{2} / \mathrm{s}$, a difference between liquidus and solidus of $100^{\circ} \mathrm{K}$, and a solidification rate of $.085 \mathrm{~mm} / \mathrm{s}$, then the gradient necessary to achieve a planar interface is approximately $2800^{\circ} \mathrm{K} / \mathrm{mm}$. Gradients of this magnitude are not typically achievable in most equipment that is available for the commercial production of single crystal turbine blades.

If, on the other hand, the solidification environment were to be controlled such that the dimensions of the dendrites are maintained at a length roughly equivalent to the diffusion boundary layer, then maximum utilization of the zone of constitutional undercooling could be achieved. Given that there is a composition gradient in advance of the solidification interface, it may be said that to a gross approximation the shortest dendrite that could be obtained can be no shorter than the diffusion boundary layer thickness. If an equivalent diffusion boundary layer thickness, $\delta_{\mathrm{C}}$, is chosen such it has a constant concentration gradient and the same total solute content as the infinite layer expressed by the exponential. Then the diffusion boundary layer thickness, $\delta_{c}$, is given by:

$$
\delta_{c}=\frac{2 D}{V}
$$

If the assumption is made that the dendrite tips are at the liquidus temperature and that the roots are at the solidus temperature of the alloy, then the gradient may be expressed as:

$$
G=\frac{\left(T_{L\left(c_{0}\right)}-T_{s\left(c_{0}\right)}\right)}{\delta_{c}}
$$

Substituting in (11) yields:

$$
G=\frac{V\left(T_{L\left(C_{0}\right)}-T_{S\left(C_{0}\right)}\right)}{2 D}
$$

which is but a factor of 2 different than the planar interface solution of (9). Evaluation of (13) with the same physical parameters as before requires a gradient in the neighborhood of $1400^{\circ} \mathrm{K} / \mathrm{mm}$, which again represents a somewhat challenging proposition. If a more reasonable gradient typical of production equipment where used to evaluate (13) the results are not acceptable. For example, if a gradient of $10^{\circ} \mathrm{K} / \mathrm{mm}$ were assumed, a typical part would require 36 hours to produce. Similar parts are regularly produced in today's production equipment in about 1.5 hours.

In production, $G / R$ techniques are limited by many of the same considerations as constant velocity techniques. In particular, material limitations preclude any significant variation in temperature and as such $G$ is difficult to control. Further, while $\mathrm{R}$ may be effected as a result of changes in the withdrawal velocity, it will be shown that holding $G / R$ constant results in a variation in withdrawal rate which is opposite to that required to produce a consistent microstructure.

Given that $G / R$ control schemes are based on the assumption that the solidification can take place in an environment where the dimension of the diffusion boundary layer is significant when compared to the dendrite length, it is unlikely that any such scheme may be implemented in a production environment. A better approach would be an attempt at maintaining a constant microstructure, thus insuring a uniform heat treat response. As such, attempts at controlling the primary dendrite arm spacing would provide a better control scheme. It has been shown ${ }^{11}$, and verified experimentally ${ }^{12}$ that the PDAS follows:

$$
\lambda_{p}=K_{p} G^{-.5} V^{-.25}
$$

Where $\lambda_{p}$ is the primary dendrite arm spacing, $K_{p}$ is a correlation constant which is based upon the solidification characteristics of the alloy, $G$ is the gradient, and $V$ is the solidification velocity. This equation implies that a more 
effective control scheme, applicable to a production environment, could be achieved through a control algorithm of the form:

$$
K=G^{2} V
$$

It should be noted that using this criteria, a decrease in $G$ would be met with an increase in $V$, a result which is exactly opposite to the $G / R$ case. No reported attempts at such a control scheme have been found.

\section{Constant Velocity Techniques}

Within some foundries, the production of single crystal turbine components may include several erroneous assumptions. The first of these is the assumption that the solidification interface is located at the baffle between the hot and cold zone of the furnace. The fallacy of such an assumption is easily understood.

Within the vacuum environment of a single crystal furnace, the predominant heat transfer mode is emissive. Given a $\Delta \mathrm{T}$ between the heat source and sink, the flux will be proportional to $\Delta T^{4}$. The total flux is dependent on the surface areas of the radiator and sink. In production furnaces the mold heater temperature and area are constant. In a similar fashion, the cold zone temperature and area are constant. However, the surface area of the part residing within the hot and cold zone respectively, changes throughout the withdrawal process ${ }^{13}$. Given that a flux balance must exist, and considering the very much greater $\Delta \mathrm{T}$ which exists below the baffle, then as a greater surface area is pulled below the baffle a commensurate increase in flux into the part must occur above the baffle. Such an increase may only be accommodated by a decrease in the average temperature of the part of the mold that is above the baffle. This has several ramifications and has been born out with finite element modeling utilizing an emissive heat transfer code called "Coyote" developed by Dave Gartling of Sandia National Laboratories, Division 1511.

First, consider that there is a heat flux through the part. This flux results in a temperature gradient across the part. The top of the part is held at a temperature which is roughly that of the mold heater. Therefore, the only manner in which the average temperature of that portion of the mold which is above the baffle may be lowered manifests itself as a decrease in the mold temperature above the baffle. Associated with this change in temperature must be a variation of the location of the solidification interface relative to the baffle. Therefore, under conditions of constant withdrawal rate, the location of the solidification interface moves up in the furnace relative to the baffle. In addition, due to the $\Delta \mathrm{T}^{4}$ nature of emissive heat transfer, this action results in an ever increasing flux into the portion of the mold below the solidification interface. Since the maximum flux through the part occurs at the baffle, the motion of the interface away from the baffle results in a situation where an ever increasing percentage of that flux is entering the part below the solidification interface, and therefore, the gradient associated with the solidification environment continuously decreases, and as a result so does the PDAS within the part. This has been verified both experimentally and with the model.

Unfortunately material limitations associated with the mold and the vaporization of volatile alloying constituents, precludes an increase in the temperature of the furnace hot zone. As a result increasing the heat flux through an increase in the $\Delta \mathrm{T}$ of the hot portion of the mold is not feasible.

\section{Process Considerations}

As described above, the solidification interface moves as a result of the changing emissive surface areas above and below the baffle. The motion of this interface away from the baffle not only serves to decrease the solidification gradicnt, but also results in a curved morphology at the solidification interface. This curvature is the result of non vertical components of the heat flux and is best understood by considering the case of a cylindrical bar.

\section{Interface Morphology}

A cylindrical bar, solidified within a standard furnace environment will have a solidification interface whose position changes as the withdrawal progress progresses. Initially the interface may be driven slightly below the baffle, however ultimately, the interface will reside above the baffle. Symmetry suggest that no matter what the location of the solidification interface to the baffle, the heat flux at the center of the bar must be strictly vertical. However, if the surface temperature of the bar differs from that of the integrated emissive view, then a horizontal component of heat flux must exist at the part surface. Therefore, at all locations except the baffle, flux lines must exhibit an increasing curvature as a function of the distance from the center of the part. As a result, an isothermal plot would show curved isotherms. This then implies that as the solidification interface moves away from the baffle, it develops a curved morphology ${ }^{14}$. If the interface is below the baffle, the center of the part is hotter than the edge and the interface is concave. Similarly, if the interface is above the baffle, then the center of the part is cooler and the interface is convex. It should be noted that such curvature has been predicted by the model and experimentally verified with BEKP (to be described later) on the solidified parts.

Since the crystallographic growth occurs against flux lines and perpendicular to the isotherms, it should be apparent that the crystallographic misorientation, which is commonly seen to increase as the process progresses, is an indication that the interface has moved and become curved. Further, visualization of these interface curvatures leads one to suspect that when the interface is driven below the baffle, sliver grains ${ }^{15}$ are more likely to nucleate, whereas when the interface is above the baffle and the gradients are dropping, dendrite tips which are broken off as a result of solute convection jets 16 are more likely to impact the mold wall and stabilize, resulting in freckle chains ${ }^{17}$.

\section{Process Improvement}

The previous discussions are intended to illustrate that there is room for improvement in many current practices for the production of single crystal turbine components. 
The process occurs under conditions where $G / R$ arguments are questionable and the process is driven by the cooling. The changing geometrical view factors can be shown to result in an increase in PDAS, growth misalignment, and the potential for an increased incidence of defects. The most obvious solution to these concerns is to find a mechanism whereby the solidification interface may be maintained at the baffle. This objective could be accomplished by reducing the effectiveness of the cold portion of the furnace. Such a reduction could be accomplished by a movable reflective insulator, used to shield the chill spool as the area for emissive heat input above the baffle decreases. Alternatively the addition of an additional heat flux to the system could serve to stabilize the location of the solidification interface; however, as previously mentioned, significant increases in the mold heater temperature are not an option. Fortunately, there is contained within the process, a source of heat which may be utilized.

\section{Utilization of Heat of Fusion}

Consider a test bar, $1.25 \mathrm{~cm}$ in diameter. If this test bar is solidifying under a gradient of $10^{\circ} \mathrm{K} / \mathrm{cm}$ and has properties similar to those of Nickel with a thermal conductivity of 1.58 Watts $/ \mathrm{cm}{ }^{\circ} \mathrm{K}$ then the heat through the part is roughly 19.4 watts. If the bar is solidifying at a rate of $.006 \mathrm{~cm} / \mathrm{s}$ and has a heat of fusion of $71.5 \mathrm{cal} / \mathrm{gm}$ and a liquid density of $7.9 \mathrm{gm} / \mathrm{cc}$ then the heat released as a result of solidification is approximately $4.2 \mathrm{cal} / \mathrm{sec}$ or 17.5 watts. Clearly the heat of solidification (17.5 watts), when compared to the heat necessary to produce the gradient (19.4 watts), is significant. The implication is that heat of fusion may be used as an internal heat source for the stabilization of the solidification environment.

\section{Experimental Procedure}

The test bar molds utilized for these experiments were provided by Rolls Royce. Furnace parameters were recorded throughout the process. Each of the ten different molds used for these trials was comprised of 8 individual test bars. Among the parameters varied were, mold preheat temperature, pour temperature and withdrawal mode (closed loop or constant velocity). For this paper, only the effects of withdrawal mode, and their implications on process control will be discussed. On each mold, one test bar included thermocouple wells which allowed junction placement at the center of the test bar. This feature also insured accurate knowledge of the location of the iunction. In this fashion, the test bars were instrumented with as many as 12 thermocouples. Preliminary work indicated that all of the test bars on a given mold were subjected to the same solidification conditions. Subsequent to the casting and growth, representative test bars from each mold were sectioned into a total of 287 transverse samples. Each sample was polished, then etched and a digital optical micrograph was obtained. The location along the test bar from which the polished samples were taken was carefully recorded. Image analysis software was developed to automatically count dendrites and determine the average primary dendrite arm spacing. Due to the direct impact on heat treatment response and the desire to evaluate solidification conditions during the process, primary dendrite arm spacing measurements were chosen as a metric of process response and overall quality.

\section{Image Analysis}

In order to accurately determine the dendrite statistics, a computer program was written to process the digital images and determine the location of the dendrite cores. Once these locations were established, it became a simple matter to ascertain the dendrite statistics. The digital images were stored as a $640 \times 480$ array of 8 bit gray scale pixels. These images were read into memory and stored as 16 bit integer numbers for ease of processing. The value of the individual pixels represented the brightness of the image. As there was a sharp demarcation in brightness between the dendritic and interdendritic regions it was not difficult to differentiate between the two types of material.

CMSX-4, the alloy used for these experiments, is cubic in nature. The cubic structure is apparent in the crosssectional micrographs of the test bars. In many of the test bars, the primary dendrites are aligned into fairly regular arrays. If rectangular sampling areas are used, this regular alignment of primary dendrites, when coupled with the dendrite spacing calculation, brings up the possibility of aliasing. Aliasing occurs when the boundaries of the sampling area are not exactly equivalent to an integral number of dendrite spacings. The result of this situation is realized by envisioning a rectangular area sliding across a regular array of dendrites. A some point, a new column of dendrites will slide into the sampling area. However, if a column does not simultaneously slide out the other side, then the number of dendrites within the sampling area has increased. This occurs despite the fact that the test sample maintains a constant dendrite spacing. The same possibility occurs in the case of vertical displacement of the sampling area. As such, aliasing can contribute to significant errors in the calculation of PDAS and the question of how to determine the spacing of primary dendrite arms needed to be addressed. However, aliasing concerns may be eliminated by uitilizing a circular sampling area.

The method used to calculate the primary dendrite arm spacing allocates each dendrite counted an equivalent area of image. As the crystal is cubic, the area allocated to each dendrite is assumed to be in the shape of a square. Since each dendrite is allocated a square area, then if the dendrites were in a regular array the distance between them would be the square root of the allocated area. The circular sampling area chosen is 464 pixels in diameter (D) and is in the center of the image. The diameter of the circle is then converted into microns using the image scale factor $(S)$. The number dendrites within this circle (N) is determined and the area of the circle (A) is calculated. The primary dendrite arms spacing $\mu$, is then calculated using equation (16).

$$
\mu=\sqrt{\frac{\pi(D S)^{2}}{4 N}}
$$

The counting software was written under the assumption that the vertical and horizontal axes on the computer 
screen would correspond to the $<001>$ directions of the secondary dendrite arms. This was not a requirement, but when combined with some care in the photography, allowed significant simplification of the dendrite recognition routine.

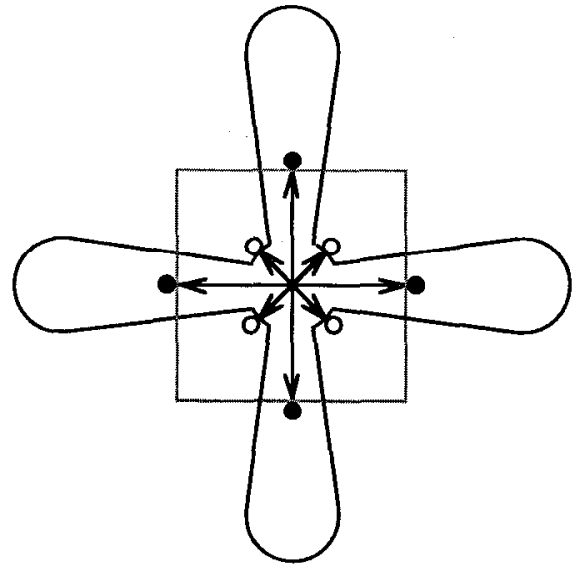

Figure 2: Dendrite Location Algorithm

Figure 2 is an illustration of a primary dendrite which will be used to describe the dendrite counting software. The program scans the rows of pixels sequentially from left to right, top to bottom. The software checks to see if the pixel currently addressed is within the sample circle, if it is not, the next pixel is checked. If the current pixel is within the sample circle then the image is interrogated at four points, each located a fixed displacement along the directions of the secondary arms. These points are shown in figure 2 as filled circles and are located at the points where the horizontal and vertical arrows impinge on the dashed square. If any of these four points possesses a brightness that is less than the brightness threshold for a dendrite, the program steps to the next pixel in the row. If all four points are brighter than the dendrite brightness level, then the software scans from the left point to the right and from the top point to the bottom. If at any time during these scans a pixel is detected with a brightness level that is lower than the dendrite brightness threshold, the program steps to the next pixel in the row. After the above procedure has occurred, the software then proceeds to verify if the location is a dendrite core. To accomplish this the program begins at the current pixel and tests outward at four points at angles $45^{\circ}$ between the secondary arm directions. These point are indicated in figure 2 as open circles. The distance from the current pixel to these points is an adjustable parameter and may be entered by the user or automatically determined by the software. If all of these four points are of a brightness that is lower than the interdentritic brightness threshold, then the current pixel is considered to be near the core of a dendrite.

Once a potential dendrite location has been identified, four diagonal scans are made and the distance from the center dendritic material to the interdendritic boundary is determined for each scan. From these four distances an average distance is calculated. The average distance is then subtracted from the actual distances and each of these four results are squared and then summed together. If the current pixel is considered to be the center of the dendrite core, then the resultant number is a measure of the asymmetry of the dendrite.

The location of the current pixel is then compared to any dendrites which may have been found previously. If the dendrite core as specified by the current pixel is within twice the primary search distance (twice the length of a side of the dashed rectangle of figure 2), of a dendrite which was found previously, then the two are considered to be the same dendrite. In such a situation, the asymmetry of the dendrites at the two core locations are compared and the location and asymmetry value of the core location with the lowest asymmetry is retained within the list of found dendrites.

After the computer has found all locations which match the selection criteria of a dendrite core, the user is then able to add or subtract additional locations. This feature proved useful, but in general, the errors made by the computer were on the order of one to two percent of the total count.

Once all dendrite locations are identified, the statistics are calculated. The primary dendrite arm spacing is determined as previously described. In addition the standard deviation in the primary dendrite arms spacing is also calculated.

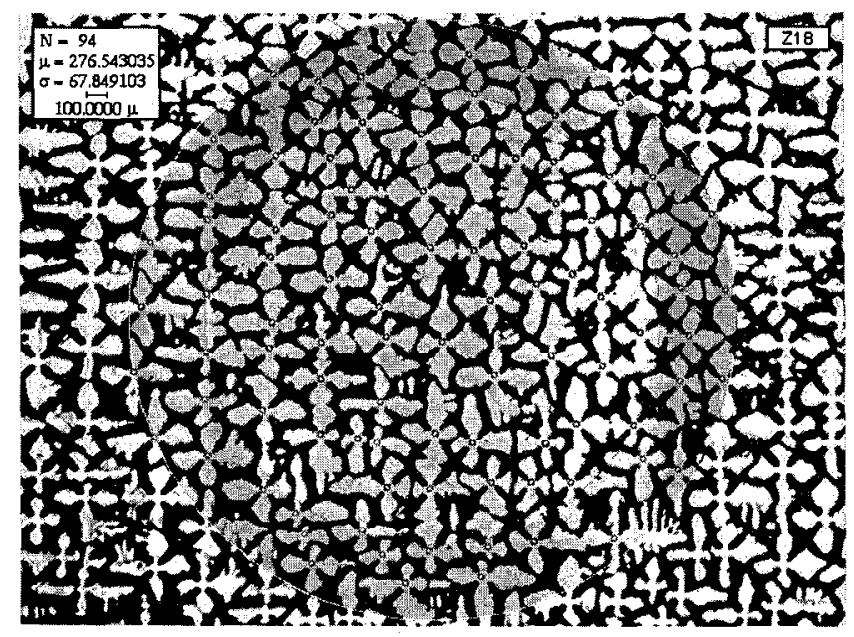

Figure 3: Example

Figure 3 is an example of a processed micrograph. The dendrite cores, as located by the software, are indicated by small circles. The identifier in the upper right hand corner identifies the test bar, in this case " $Z$ " and the slice number, "18". The box in the upper left hand corner displays the number of dendrites " $N$ ", the primary dendrite arm spacing " $\mu$ ", the standard deviation in the spacing $\sigma$, and a micron marker. This date was additionally written to a file for subsequent processing.

\section{Data Reduction}

Given the temperature and location of each thermocouple along the part, least squares techniques can be used to generate equations for both temperature and location, each as a function of the other. In this fashion, thermocouple data was processed continuously throughout each withdraw cycle yielding equations in the form of (17) and (18) below. 


$$
\begin{aligned}
& T=f^{3}(x) \\
& x=f^{3}(T)
\end{aligned}
$$

Thermocouples with values nearest the solidification temperature were weighted more heavily than those with temperatures farther away from the solidification band. This procedure increased the confidence that the resultant curve was representative throughout the solidification region. Once equation (17) had been obtained, it was differentiated with respect to $x$; the result being equation (19), an expression for the temperature gradient as a function of location along the part.

$$
G=\frac{\partial T}{\partial x}=\frac{\partial\left(f^{3}(x)\right)}{\partial x}
$$

Due to the dynamic nature of the solidification process, these equations were reformulated several times a minute. Given (18) and (19), a real time measure of the solidification environment is possible.

In order to characterize the solidification conditions, a temperature, chosen to be half way between the liquidus and solidus, is entered into equation (18). The result is the position along the test bar of the center of the solidification band. This location, when combined with the distance that the part has been withdrawn, allows the determination of the location of the solidification interface with respect to the baffle. The location obtained from (18) is also used in conjunction with (19) to obtain the gradient at the solidification interface.

These data were combined with the dendrite measurements to provide information regarding the PDAS and the associated gradient and solidification rate for each slice.

Under conditions of constant withdrawal velocity, data analysis revealed that the motion and characteristics of the solidification interface vary significantly throughout the process. In addition to the interface position, the gradient within the part, around the solidification interface, was also seen to change during the growth process. Analysis of the thermocouple data revealed that the solidification gradient is a function of the position of the solidification interface relative to the baffle. Further, the motion of the solidification interface within the furnace environment results in solidification rates which vary as well. Hence, within this process, the physical environment results in solidification conditions which become a location dependent function. When solidification conditions for each slice (as expressed by $1 / G^{5} R^{25}$ ) are plotted vs. the measured primary dendrite arm spacing (PDAS), a best fit equation (20) results.

$$
\text { PDAS }=231+\frac{330}{G^{.5} R^{.25}}
$$

Utilizing (20) and the gradient and rate information obtained from the thermocouple data as well as the withdrawal position, it is possible to predict the PDAS as a function of position along the test bar. Post procesing of the thermocouple and withdrawal position data resulted in 5th order polynomials for both the solidification gradiant and solidification rate as a function of position along the bar. These equations were substituted into (20) for $G \& R$ an the results are plotted in figure 4 . (It should be noted that for these experiments a nominal pour temperatures of $1500^{\circ} \mathrm{C}$ and a susceptor temperatures of $1475^{\circ} \mathrm{C}$ were utilized.)

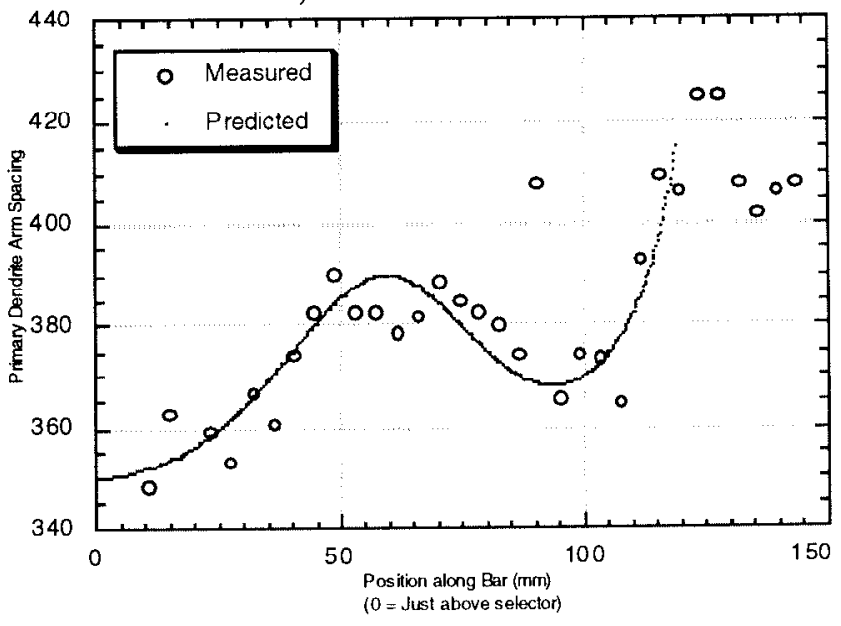

Figure 4: Measured and Predicted PDAS as a Function of Test Bar Position.

Figure 4 exhibits a good correlation between the calculated and experimental PDAS data and indicates that the relationship for PDAS as expressed in (14) is in fact valid. It may therefore be inferred that there exists a set of measurable furnace parameters capable of predicting at a minimum, the variation in PDAS that may be occurring during the process. This data also implies that thermocouple data may be effectively used as a means of determining the location of the solidification interface and solidification rate in real time.

It should be noted that the peak in PDAS occurs at the point where the solidification interface moved to its "setpoint position" of $7 \mathrm{~mm}$ above the baffle. The subsequent reduction in PDAS is due to the controller action. The final increase in PDAS occurs as a result of the solidification process moving beyond the usefulness of the thermocouple array. Optimization and improved process tuning should mitigate this effect.

\section{Interface Morphology Measurements}

As previously stated, variations in solidification conditions are due to the changing emissive environment that occurs as the ratio of mold surface areas exposed to heating and cooling conditions changes. This results in varying heat fluxes across the solidification interface and as the process progresses, promotes the motion of the solidification interface up into the hot zone of the furnace.

As the solidification interface moves away from the baffle between the hot and cold zone of the furnace, it develops a curved morphology due to the increasing horizontal components of the heat flux. This curved interface morphology results in a variation of the crystallographic orientation of the part and, in severe cases, is cause for rejection. Such curvature was verified using Backscattered Electron Kikuchi Patterns (BEKP) in the SEM. These patterns arise when the electron beam within an SEM is focused on a single spot. Electrons, which are reflected 
back out of the surface, form diffraction patterns characteristic of the crystalline structure and its orientation. BEKPs were used to chart the orientation shift which occurred across the solidified test bar sections. Since the preferred growth is in the $\left\langle\begin{array}{llll}0 & 0 & 1\end{array}\right\rangle$ direction, and dendrite growth is perpendicular to the solidification isotherms, then by measuring the angle that the $<0 \quad 0 \quad 1>$ crystallographic direction makes with the sample surface, the shape of the solidification interface is revealed (assuming steady state conditions). Such measurements revealed both a curved interface and an asymmetry that was probably due to the uneven emissive environment that results from the presence of the mold tree. The degree of curvature was also seen to increase as the process progressed. As mentioned, the combination of this curvature and extended dendrites which may occur as a result of the lower gradients could be responsible for the formation of freckle chains and serve as an additional driving force for the development of real time process control.

\section{Process Stabilization}

In order to gain better control of the process, it was proposed that an increase in the withdrawal rate, must be (to a point) balanced by an increase in the solidification rate. This action results in an associated increase in the heat released as a result of solidification. This heat is sufficient to change the fluxes through the part and thereby lower the position of the solidification interface while increasing the gradient. These coupled phenomena may be effectively used as a method of microstructural stabilization in a closed loop control scheme while simultaneously improving crystallographic orientation.

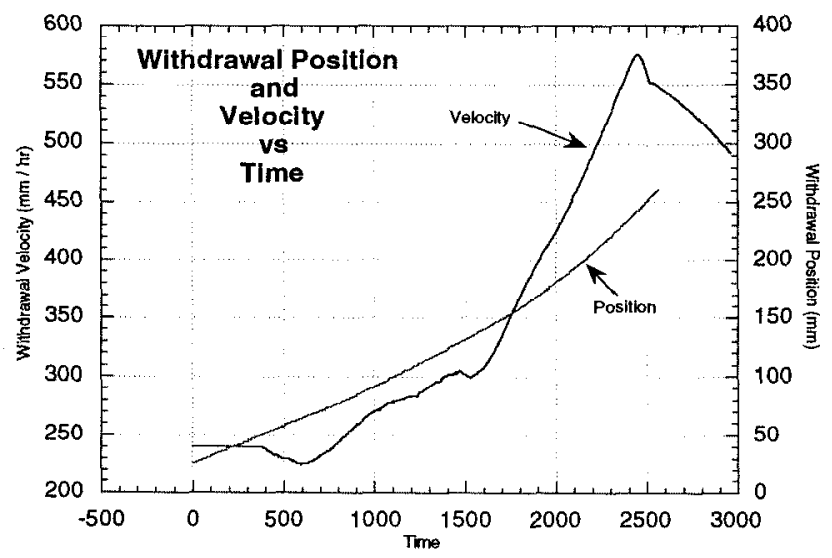

Figure 5: Closed Loop Control Response

For the closed loop process trial illustrated in Figure 5, a technique was developed which maintained the position of the solidification interface at the baffle. Utilizing the derived least squares polynomial equation for position as a function of temperature (18), and coupling it with the withdrawal distance, the location of the solidification interface relative to the baffle may be determined. During the growth process, the velocity was continuously adjusted in an attempt to maintain the center of solidification band at the baffle. In this fashion, an increase in the solidification rate resulted in enough excess heat to effectively drive the equilibrium position of the solidification interface down into regions of higher gradient and more vertical heat flux. Such a scheme ensures that the solidification zone is maintained at the highest achievable gradient within the available thermal environment. As may be seen from the figure, the technique results in an ever increasing withdrawal velocity. Such a result is expected, as the heat released on solidification must be of sufficient magnitude to overcome the heat losses associated with an ever increasing emissive radiator below the baffle. It is of interest to note that the final velocity utilized is well beyond that which is typically employed for this process, and yet good crystals were achieved.

Although this method does not explicitly control primary dendrite arm spacing, it does provide for a flatter interface which will result in fewer orientation defects. Further, utilization of this technique does result in solidification conditions which begin to approximate the previously mentioned $\mathrm{K}=\mathrm{G}^{2} \mathrm{~V}$ condition. In addition, the positioning of the solidification zone at the location of highest gradient should minimize those defects which may be associated with a low gradient environment. It may therefore be inferred that by utilizing the heat of fusion as an internal heat source, the effect of increasing cooling is offset and a more regular microstructure should result. Experimental results reveal that these expectations are in fact valid.

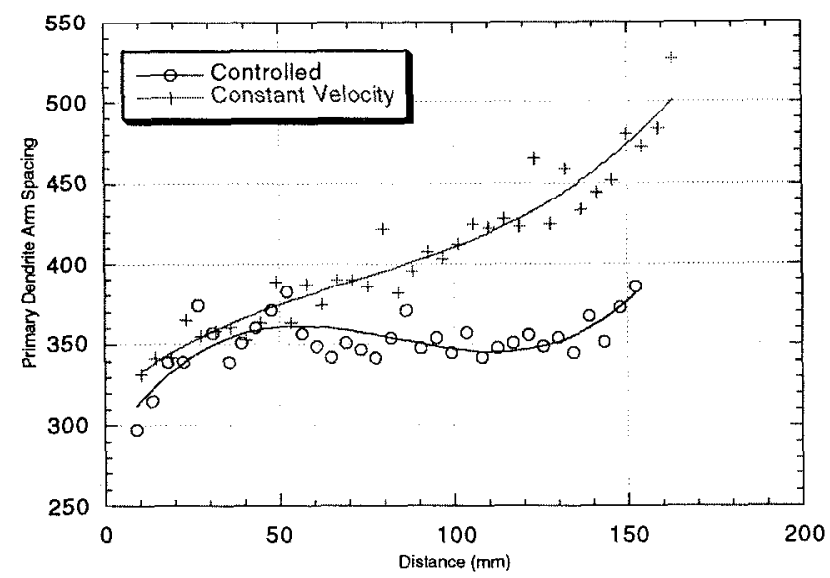

Figure 6: PDAS Measurements for Constant Velocity \& Closed Loop Processing vs. Test Bar Location

Figure 6 is a comparison of the PDAS results for two process runs. The associated test bars utilized alloy from the same heat and in the same furnace, with identical pour rates, metal pour temperatures, and mold heater temperatures. Both molds were initially processed at a withdrawal rate of $240 \mathrm{~mm} / \mathrm{hr}$. However, during the closed loop trial a computer varied the withdrawal rate as described above and illustrated in figure 5. As Figure 6 demonstrates, a significant stabilization of the PDAS may be achieved as a result of real time closed loop control techniques. 


\section{Conclusion}

It has been shown that the changing emissive environment within today's production single crystal furnaces results in a gradient of decreasing magnitude that moves away from the baffle as the withdrawal process progresses. It has been additionally demonstrated that the heat of fusion may be used as an internal heat source to counteract this effect. As such, and contrary to published $G / R$ schemes, the decrease in gradient which occurs as the process progresses should be counteracted will an increase in withdrawal rate. Such a rate increase is consistent with the expression for primary dendrite arm spacing:

$$
\lambda_{p}=K_{p} G^{-.5} V^{-.25}
$$

and experimental results have demonstrated that this results in a stabilization of the microstructure. Commensurate with the microstructural stabilization is a flattening of the solidification interface morphology which occurs as a result of the more vertical heat flux in the vicinity of the baffle. This condition results in a reduction of orientation misalignment between the <001> and the stress axis of the part. Finally, these conditions should reduce defects such as freckles by maintaining the optimum solidification environment achievable for the configuration.

In practice, a fully instrumented mold would be subjected to a closed loop scheme which maintains the solidification interface at the baffle. The resultant velocity profile would be recorded and played back for noninstrumented parts. It is expecled that final velocities may be well above what is normally considered prudent.

As a side bar, it should be noted that an increase in the amount of metal above the useful portion of the part can to some degree mitigate the effect of increased cooling. The flip side of this statement is that cost reduction efforts associated with reduced pour volumes may have a negative impact on process yields unless process modifications, such as those described herein, are implemented.

\section{Acknowledgments}

This work is supported by the U.S. Department of Energy under contract number DE-AC04-94AL85000.

The author would like to acknowledge Retech Inc. and Rolls Royce for their support in this effort. In particular Alan Moulden, Alan Patrick and Ray Snider of Rolls Royce are recognized for their support and insight, Thanks are also extended to Joe Michael and Randy Schunk of Sandia National Labs for their support and assistance with the BEKP and modeling efforts respectively.

1 M.E. Schlienger, "Understanding and Development of Advanced Techniques for the Processing of Single Crystal Turbine Components", (Ph.D. Thesis, Oregon Graduate Institute of Science and Technology, February 1995).
2 VerSnyder, F.L. and Guard, R.W., "Directional Grain Structures fort High Temperature Strength", ASM Transactions, 52, 1959.

3 Erickson, J.S., Oxczarski, W.A. and Curran, M .C., "Process Speeds Up Directional Solidification", Metal Progress, March, (1971)

4. Higginbothom, G.J.S, "From Research to Cost Effective Directional Solidification and Single Crystal Production - An Integrated Approach", Materials and Design, 8 (1987)

5 Cole, G.S. and Cremesio R.S., "Solidification and Structure Control in Superalloys", The Superalloys, (New York, NY: John Wiley and Sons, 1972) 479-508.

$6 \quad$ Staub, F. and Walser B., "An Alternative Process for the Manufacture of Single Crystal Gas Turbine Blades", Sulzer Technical Review, March, (1988), 11-16.

7 Yoshinari A., Morimoto s. and Kodama H., "Single Crystal Growth Technology of Nickel Base Alloys", Proceedings 1st Intl. SAMPE Symposium, Nov 28, - Dec 1, 1989

8 Nakagawa Y.G. "Development of Solidification Technology for Superalloys in Japan" Superalloys (1985) Japan-US Seminar

9 Kurz W. and Fisher D.: Fundamentals of Solidification, (Aedermannsdorf, Switzerland, Trans Tech Publications, 1986), 157

10 Kurz W. and Fisher D.: Fundamentals of Solidification, (Aedermannsdorf, Switzerland, Trans Tech Publications, 1986), 192

11 Kurz W. and Fisher D.: Fundamentals of Solidification, (Aedermannsdorf, Switzerland, Trans Tech Publications, 1986), 213

12 Wills V.A. and McCartney D.G. "A Comparative Study of Solidification Features in Nickel-Base Superalloys: Microstructural Evolution and Microsegregation", Mat. Sci. and Eng. A145(1991), 223-232.

13 Hediger F. and Sahm, P.R., "Simulation of DS and SC Processes and Their Experimental Verification", Modeling of Casting and Welding Processes IV, (Warrendale, PA, TMS Publications, 1988), 645-657.

14 Morimoto, S., Yoshinari, A., Niyama, E., "Effects of Thermal Variables on the Growth of Single Crystals of NiBase Superalloys", Superalloys 1984, (Warrendale, PA, TMS Publications, 1984), 177-184

15 Pollock T.M., et al. "Grain Defect Formation During Directional Solidification of Superalloys", Superalloys 1992, (Warrendale, PA, TMS Publications, 1992), 125 - 134

${ }_{16}$ Copley, S.M., et al., "The Origin of Freckles in Unidirectionally Solidified Castings", Metallurgical Transactions, 1(8), (1970), 2193-2204.

17 Giamei, A.F. and Kear, B.H., "On the Nature of Freckles in Nickel-Base Superalloys", Metallurgical Transactions, 1(8), (1970), 2185-2192. 